九州大学学術情報リポジトリ

Kyushu University Institutional Repository

\title{
Oral administration of l-serine reduces the locomotor activity of socially isolated rats
}

\section{Shigemi, Kazutaka}

Laboratory of Regulation in Metabolism and Behavior, Graduate School of Bioresources and Bioenvironmental Sciences, Kyushu University

Tsuneyoshi, Yousuke

Laboratory of Regulation in Metabolism and Behavior, Graduate School of Bioresources and Bioenvironmental Sciences, Kyushu University

\section{Yamada, Satoshi}

Laboratory of Regulation in Metabolism and Behavior, Graduate School of Bioresources and Bioenvironmental Sciences, Kyushu University

Kabuki, Yusuke

Laboratory of Regulation in Metabolism and Behavior, Graduate School of Bioresources and Bioenvironmental Sciences, Kyushu University

他

http://hdl. handle. net/2324/26474

出版情報：Neuroscience Letters. 468 (1)，pp.75-79，2010-01-01. Elsevier バージョン：

権利関係 : (C) 2009 Elsevier Ireland Ltd. 
Oral administration of L-serine reduces the locomotor activity of socially isolated rats

Kazutaka Shigemi, Yousuke Tsuneyoshi, Satoshi Yamada, Yusuke Kabuki, Kohsuke Hayamizu ${ }^{a}$, D. Michael Denbow ${ }^{b}$, Mitsuhiro Furuse

Laboratory of Regulation in Metabolism and Behavior, Graduate School of Bioresources and Bioenvironmental Sciences, Kyushu University, Fukuoka 812-8581, Japan

${ }^{a}$ FANCL Research Institute, 12-13 Kamishinano, Totsuka-ku, Yokohama, Kanagawa, 244-0806, Japan

b Department of Animal and Poultry Sciences, Virginia Polytechnic Institute and State University, Blacksburg, VA 24061-0306, USA

The number of text pages (including figures): 26

The number of figures: 3

\section{Correspondence should be addressed to: Mitsuhiro Furuse}

Laboratory of Regulation in Metabolism and Behavior, Graduate School of Bioresources and Bioenvironmental Sciences, Kyushu University, Fukuoka 812-8581, Japan 
Tel. (81)(92)642-2953

Fax $(81)(92) 642-2953$

E-mail: furuse@brs.kyushu-u.ac.jp

\section{Acknowledgements}

This work was supported by a Grant-in-Aid for Scientific Research from Japan Society for the Promotion of Science. 
Keywords:

L-Serine

Rat

Social isolation

Locomotor activity 


\section{Abstract}

L-Serine is considered a functional amino acid in the central nervous system, since intracerebroventricular injection of L-serine induced sedative and hypnotic effects in neonatal chicks exposed to acute stressful conditions. Accordingly, L-serine is a candidate anti-stress factor, but the effect of daily intake of L-serine on behavior of animals exposed to chronic stress has not been investigated. In the present study, we exposed rats to social isolation stress for 4 weeks, and home cage test and open field test were concluded to evaluate the effect of L-serine on behavior. To investigate L-serine supplementation modifies the brain L-serine and its metabolite contents, free amino acid contents were measured by a high performance liquid chromatography. L-Serine in the drinking water increased L-serine levels in some brain areas, but changes in its metabolites were almost negligible. L-Serine decreased locomotor activity in rats exposed to a familiar environment. In addition, L-serine decreased exploratory behavior of isolated rats, even in a novel environment. Our results could suggest that daily intake of L-serine can attenuate symptoms induced by chronic stress. 


\section{Introduction}

L-Serine biosynthesized from a glycolytic intermediate by phosphoglycerate dehydrogenase (Phgdh) is an important nutrient for brain function and development as evidenced by patients with Phgdh deficiency who suffer from severe neurological symptoms [19]. Additionally, results from Phgdh null knockout mice show that the Phgdh-dependent pathway of de novo synthesis was the principal source of L-serine in developing embryos. This pathway is essential for normal embryonic development, especially for brain morphogenesis [36]. Mitoma et al. [23] reported that L-serine increased the viability and promoted the growth of dendrites in cultured rat hippocampal neurons. These facts indicate the importance of L-serine in the central nervous system (CNS).

It has been suggested that L-serine-derived membrane lipids are required for developing neurons [17]. This idea is supported by the findings that neurons cannot maintain synthesis of serine-derived lipids without an external supply of L-serine [24, 36]. Furthermore, some research groups have demonstrated that chronic oral intake of phosphatidylserine, one of the L-serine-derived lipids, prevents an age-related reduction in dendritic spine density of CA1 pyramidal cells in the rat hippocampus [25] and improves memory impairment of aged rats [32]. On the other hand, Koutoku et al. [20] reported that intracerebroventricularly (i.c.v.) injected phosphatidylserine attenuated stress-like behavior of chicks under acute isolation-induced stress. They also revealed that L-serine as a component of 
phosphatidylserine showed a similar function as observed with phosphatidylserine. Therefore, it appears possible that dietary L-serine may affect brain function and regulate behavior.

Social isolation is thought to be stressful for normally gregarious animals. Young rats deprived of social contact with other rats experience a form of prolonged stress that leads to long-lasting alterations in their behavioral profile [28]. It has been reported that chronically isolated rats show some neurological symptoms including neophobia [12], learning and memory impairments $[4,26]$, and locomotor hyperactivity $[6,15]$. Above all, the most consistent behavioral response to isolation rearing is hyperlocomotion [22]. In the present study, therefore, we investigated whether chronic intake of L-serine can improve abnormal behavior of socially isolated rats.

\section{Materials and methods}

Male Sprague-Dawley rats, 3 weeks-of-age, were purchased from Japan SLC Inc. (Hamamatsu, Japan). To minimize animal suffering, the number of animals used was based on the minimum required for statistically valid results ( $n=7$ per group). After arrival, all rats were immediately placed in individual plastic cages $(38 \times 22 \times 8 \mathrm{~cm})$ because the full behavioral changes associated with social isolation of rodents from weaning are only observed if this intervention is commenced during a critical period (from postnatal day (PND) 20 to PND30) around the time of puberty [11]. All rats were 
allowed free access to a commercial diet (MF; Oriental Yeast, Tokyo, Japan) and drinking water. A 12 -h light/dark cycle was maintained (light on at 08:00, light off at 20:00) throughout the experiment and room temperature was maintained at $23 \pm 1^{\circ} \mathrm{C}$. The animals were subjected to experimental procedures described below. The experimental procedures followed the Guidelines for Animal Experiments of the Faculty of Agriculture and the Graduate School of Kyushu University, as well as Japanese Law (No. 105) and a Notification (No. 6) by the Japanese Government.

After a 5 day acclimation period, locomotor activity of rats in their home cages was measured and then they were divided in three groups so that their average values of locomotor activity were similar. The three treatment groups consisted of rats provided either distilled water (control), $0.2 \%$ L-serine (Kyowa Hakko Bio, Tokyo, Japan) in distilled water, or $2 \%$ L-serine in distilled water. The water treatments continued till the conclusion of the experiment.

Locomotor activity in the home cages was measured during the $12 \mathrm{~h}$ dark period at 1,2 and 3 weeks after the onset of L-serine treatment. The activity was counted with an infrared beam sensor (NS-AS01: Neuroscience Inc., Tokyo, Japan) placed about $8 \mathrm{~cm}$ above the center of the cage, and analyzed using the software DAS-008 (Neuroscience Inc.).

Eleven days after the final test in their home cages, locomotor activity in a novel environment was recorded employing the open field test. Briefly, animals were individually transferred to an open field arena. The arena was 
circular (diameter $60 \mathrm{~cm}$ and height $35 \mathrm{~cm}$ ) and made of black takiflex. The test was begun by placing individual animals at the center of the arena and the behavior of animals was then observed for 30 min under 100 lux illumination. After each test, the field was cleaned with a $70 \%$ ethanol solution. The following behavioral categories were examined: path distance, time spent moving, speed of movement and frequency of rearing behavior. All behaviors except rearing were automatically analyzed with a computer-based video tracking system (AXIS-90; Neuroscience Inc., Tokyo, Japan). Frequency of rearing behavior was manually recorded.

At 3 days after the open field test, rats were decapitated under anesthesia with pentobarbital sodium and the brain samples (cerebral cortex and hippocampus) collected. Samples were immediately frozen at $-80^{\circ} \mathrm{C}$ until analyzed.

The concentrations of free amino acids were analyzed by high performance liquid chromatography (HPLC). The tissue samples were homogenized in ice-cold $0.2 \mathrm{M}$ perchloric acid solution containing $0.01 \mathrm{mM}$ EDTA $2 \mathrm{Na}$ and left for deproteinization in ice. After $30 \mathrm{~min}$, the mixtures were centrifuged at $20,000 \mathrm{x}$ for $15 \mathrm{~min}$ at $0^{\circ} \mathrm{C}$ and the resultant supernatants then adjusted to pH 7 with 1 M sodium hydroxide. Each sample $(20 \mu 1)$ was then completely dried under reduced pressure. Dried residues were dissolved in $10 \mu \mathrm{l}$ of $1 \mathrm{M}$ sodium acetate-methanol-triethylamine (2:2:1) solution, re-dried, and dissolved in $20 \mu 1$ of derivatization solution (methanol-water-triethylamine-phenylisothiocyanate $(7: 1: 1: 1))$. After 20 
min at room temperature, phenylisothiocyanate was allowed to react with the amino groups and the samples were dried again and then dissolved in $100 \mu l$ of Pico-tag Diluent (Waters, Milford, MA, USA). These diluted samples were filtered through a 0.45 -mm filter (Millipore, Bedford, US A). The same method was applied to standard solutions prepared by diluting a commercially available L-amino acid solution (Type AN II and Type B; Wako, Osaka, Japan) with distilled water. These derivatized samples were applied to a Waters HPLC system (Pico-tag free amino acid analys is column (3.9 $\mathrm{mm} \times 300 \mathrm{~mm})$, Alliance 2690 separation module, 2487 dual-wavelength UV detector, and Millennium 32 chromatography manager; Waters, Milford, USA). They were equilibrated with buffer A $(70 \mathrm{mM}$ sodium acetate (pH 6.45 with $10 \%$ acetic acid)-acetonitrile $(975: 25))$ and eluted with a linear gradient of buffer B (water-acetonitrile-methanol $(40: 45: 15))(0,3,6,9,40$, and $100 \%$ ) at a flow rate of $1 \mathrm{ml} / \mathrm{min}$ at $46^{\circ} \mathrm{C}$. The absorbance at $254 \mathrm{~nm}$ was applied to determine concentrations of free amino acids. Triethylamine and sodium acetate trihydrate were purchased from Wako (Osaka, Japan), while other drugs were purchased from Sigma (St Louis, USA).

Home cage tests were analyzed with repeated measure ANOVA. Open field tests and amino acid levels were analyzed with one-way ANOVA, and a Tukey-Kramer test was done as a post hoc test. Further, regression analysis between concentrations of L-serine in the cerebral cortex or in the hippocampus and path distance, time spent moving, and frequency of rearing behavior in the open field test was done, respectively. Significant 
differences implied $\mathrm{P}<0.05$. Values are presented as means \pm S.E.M. Data were analyzed using the statistical program StatView Version 5.0 (SAS Institute, Cary, US A, 1998).

\section{Results}

Total L-serine intake over the experimental period was $2.00 \pm 0.02 \mathrm{~g}$ and $14.38 \pm 0.76 \mathrm{~g}$ in the $0.2 \%$ and $2 \%$ L-serine groups, respectively. Fig. 1 shows the effect of L-serine on locomotor activity of rats in their home cages during the dark period. L-Serine significantly $[\mathrm{F}(2,18)=4.558, \mathrm{P}<0.05]$ decreased the locomotor activity of rats which significantly $[F(3,54)=$ $20.453, \mathrm{P}<0.0001]$ increased over time. There was no significant $[\mathrm{F}(6,54)$ $=1.425, \mathrm{P}>0.05]$ interaction between $\mathrm{L}-$ serine treatment and time.

Results for the open field test are shown in Fig. 2. While L-serine decreased the distance traveled by rats in the arena, this effect was not significant $[\mathrm{F}(2,18)=2.530, \mathrm{P}>0.05]$. The spent time in the arena $[\mathrm{F}(2,18)$ $=2.486, \mathrm{P}>0.05]$ and their movement speed $[\mathrm{F}(2,18)=0.873, \mathrm{P}>0.05]$ were not significant. However, L-serine significantly $[\mathrm{F}(2,18)=4.108, \mathrm{P}<0.05]$ decreased the frequency of rearing behavior.

The concentrations of free amino acids that changed due to L-serine treatment are shown in Fig. 3. Administration of L-serine in the water increased $[\mathrm{F}(2,18)=9.442, \mathrm{P}<0.005]$ the concentrations of L-serine and L-cystathionine $[\mathrm{F}(2,18)=5.074, \mathrm{P}<0.05]$ in the cerebral cortex. Furthermore, the concentration of L-serine was significantly $[\mathrm{F}(2,17)=$ 
5.154, P $<0.05]$ increased in the hippocampus. Although L-cystathionine tended to be increased, the effect was not significant. No significant changes were detected in the concentrations of other amino acids (glycine, L-alanine, L-aspartate, L-glutamate, etc.) derived from the L-serine metabolic pathway (data not shown).

\section{Discussion}

The so-called "isolation syndrome" has been well characterized in rats and includes spontaneous locomotor hyperactivity, enhanced response to a novel environment, learning and memory impairments, deficits in prepulse inhibition [8], and abnormal hippocampal structure [33]. This non-pharmacological animal paradigm which elicits robust, reproducible, developmental alterations is induced by preventing social contact after the weaning period. This may be an animal model for human mental disorders because similar environmental interventions during early life in humans may contribute to the development of common psychiatric disorders such as depression and schizophrenia $[11,16]$. Therefore, the rat social isolation paradigm may enable discovery and evaluation of novel therapeutic agents against mental disease. In the present study, we investigated whether L-serine affects the behavior of isolated rats.

The locomotor activity of rats in their home cages during the dark period was decreased by the addition of L-serine in the drinking water. In the open field test, L-serine decreased exploration activity of rats in the open field 
arena (evidenced by decreased frequency of rearing). Further, the concentration of L-serine was increased in the cerebral cortex and hippocampus by L-serine. The regression analysis demonstrated significant negative correlations between L-serine concentration in the cerebral cortex and behavioral parameters in the open field test. The following information is the detailed statistical data of the regression analysis. Distance of path $(\mathrm{cm})=10124\left(\right.$ S.E. 2759) $-2.02($ S.E. 0.85$) X\left(R^{2}=0.228, \mathrm{P}<0.05\right)$. Time $(\mathrm{s})$ $=462\left(\right.$ S.E. 123) $-0.091\left(\right.$ S.E. 0.038)X $\left(R^{2}=0.234, \mathrm{P}<0.05\right)$. Frequency of rearing behavior $($ count $)=72.5\left(\right.$ S.E. 22.05) $-0.015\left(\right.$ S.E. 0.007) $X\left(R^{2}=\right.$ $0.196, \mathrm{P}<0.05)$. These results suggest that L-serine accumulated in the brain passing through the blood brain barrier and changed the behavior of rats in both the familiar and novel environment.

If additional groups of rats housed in company were prepared to have a measure of basal activity in socially-housed rats, the effect of L-serine obtained here might be clearer. In our experimental design, however, rats must be measured their locomotor activity singly in their home cage. If we set a group rearing control having more than two rats, we could not measure locomotor activity in familiar environment of these groups. Our model could not confirm that the improvement by L-serine was comparable to the condition in which the rats were reared in groups. However, our major interest is whether L-serine ameliorates the stress response when compared with the rat without L-serine. According to these reasons, we have made the plan for the present study. 
Although the mechanism whereby L-serine decreased the effects of isolation stress was unclear, L-serine may act to counterbalance the biological changes induced by isolation stress. Interestingly, decreased dendritic spine density of pyramidal neurons in the prefrontal cortex and hippocampus was reported in isolated rats [30] and phosphatidylserine, one of L-serine-derived lipids, could ameliorate the dendritic spine loss of pyramidal cell in aged rats [25]. Therefore, L-serine may ameliorate the dendritic spine loss of pyramidal cell by enhancing synthesis of phosphatidylserine in isolated rats, although this has not been investigated.

On the other hand, we reported that centrally administered L-serine induced a sedative and hypnotic effect in neonatal chicks exposed to isolation stress $[1,2,20]$. These L-serine-induced behavioral changes were through the activation of $\gamma$-aminobutyric acid (GABA) A receptors [29]. The functional impairment of inhibitory GABAergic interneuron is reported as one of the neurochemical changes associated with isolated rats $[14,27]$. Furthermore, it was confirmed that diazepam $(0.5 \mathrm{mg} / \mathrm{kg}$, i.p. $)$, a $\mathrm{GABA}_{\mathrm{A}}$ receptor agonist, injected for 5 days reduced the locomotor activity of isolated mice [21]. It is possible that the results obtained here may be explained by L-serine effects on the GABAergic systems.

L-Serine increased L-cystathionine content in the cerebral cortex and had a similar tendency in the hippocampus (Fig. 3). L-Cystathionine is synthesized by condensation of L-serine with L-homocysteine in a reaction catalyzed by cystathionine $\beta$-synthase (CBS), so it seems reasonable that 
L-serine stimulated L-cystathionine synthesis by acting as a substrate for CBS in the brain of isolated rats. A polymorphism of the CBS allele is significantly underrepresented in children with a high IQ, suggesting that CBS may influence cognitive function [3]. On the other hand, a deficiency of CBS causes homocystinuria, an inborn error of metabolis m characterized by mental retardation, seizures, psychiatric disturbances, skeletal abnormalities, and decreased L-serine and glycine levels in cerebrospinal fluid $[5,10,31]$. It has been reported that oral supplementation of L-serine or glycine was useful in decreasing homocysteine levels of rats in methionine-induced hyperhomocysteinemia [13]. These phenomena resemble the events observed in L-serine deficiency syndrome suggesting the presence of a common mechanism between the two diseases. Interestingly, CBS is preferentially expressed in the radial glia/astrocyte lineage whereas it is hardly detectable in neuronal cells [9], and Phgdh is preferentially expressed in the radial glia/astrocyte lineage and olfactory ensheathing glia in mouse brain [35] suggesting that Phgdh may supply L-serine for the CBS-mediated metabolism of homocysteine in these cells. L-Cystathionine is converted to L-cysteine in a reaction catalyzed by cystathionine $\gamma$-lyase, and cysteine is the limiting reagent in the synthesis of glutathione (GSH), a major antioxidant. In fact, L-propargylglycine, a specific inhibitor of cystathionine $\gamma$-lyase, resulted in loss of GSH in the mouse brain indicating that cysteine is important for the maintenance of GSH levels in the CNS [7]. 
Unfortunately, in the present study, the content of L-cysteine could not be detected because of the overlap of its peak with L-cystine, a dimmer of L-cysteine. Increased L-cystathionine in the cerebral cortex of isolated rats in this study could suggest enhanced glutathione synthesis, although we did not assay GSH levels directly. It is reported that social isolation stress causes oxidative damage to the brain via enhanced lipid peroxidation and NO production, and the brain content of GSH was significantly decreased in socially isolated mice in an exposure period-dependent manner [18]. Therefore, L-serine supplementation could be useful to support an antioxidant system by increasing GSH in isolated rats.

On the other hand, other amino acids derived from the L-serine metabolic pathway did not change even when L-serine was applied. Asechi et al. [2] confirmed that the brain free L-serine level increased after i.c.v. injection of L-serine, but no significant changes in other amino acids were detected. They did not measure L-cystathionine levels, but L-cystathionine may be an important pathway for L-serine. At present, although it is not clear whether L-cystathionine itself functions in the brain, its metabolite L-cysteine had a similar function as found with L-serine [1,34]. The function of L-cystathionine should be clarified in the future.

In conclusion, our results could suggest that daily intake of L-serine can attenuate symptoms induced by isolation stress in rats. Here, we propose that the requirement of nonessential amino acids such as L-serine should be paid attention to carefully for the stressful society. 


\section{Acknowledgements}

This work was supported by a Grant-in-Aid for Scientific Research from Japan Society for the Promotion of Science. 


\section{References}

[1] M. Asechi, S. Tomonaga, T. Tachibana, L. Han, K. Hayamizu, D. M. Denbow, M. Furuse, Intracerebroventricular injection of L-serine analogs and derivatives induces sedative and hypnotic effects under an acute stressful condition in neonatal chicks, Behav. Brain Res. 170 (2006) $71-77$.

[2] M. Asechi, I. Kurauchi, S. Tomonaga, H. Yamane, R. Suenaga, Y. Tsuneyoshi, D. M. Denbow, M. Furuse, Relationships between the sedative and hypnotic effects of intracerebroventricular administration of L-serine and its metabolites, pyruvate and the derivative amino acids contents in the neonatal chicks under acute stressful conditions, Amino Acids 34 (2008) 55-60.

[3] S. Barbaux, R. Plomin, A. S. Whitehead, Polymorphisms of genes controlling homocysteine/folate metabolism and cognitive function, Neuroreport $11(2000)$ 1133-1136.

[4] M. Bianchi, K. F. C. Fone, N. Azmi, C. A. Heidbreder, J. J. Hagan, C. A. Marsden, Isolation rearing induces recognition memory deficits accompanied by cytoskeletal alterations in rat hippocampus, Eur. J. Neurosci. 24 (2006) 2894-2902.

[5] N. A. J. Carson, D. W. Neill, Metabolic abnormalities detected in a survey of mentally backward individuals in northern Ireland, Arch. Dis. Child. 37 (1962) 505-513.

[6] A. Del Arco, S. Zhu, A. Terasmaa, A. H. Mohammed, K. Fuxe, 
Hyperactivity to novelty induced by social isolation is not correlated with changes in D2 receptor function and binding in striatum, Psychopharmacology(Berl.) 171 (2004) $148-155$.

[7] L. Diwakar, V. Ravindranath, Inhibition of cystathionine- $\gamma$-lyase leads to loss of glutathione and aggravation of mitochondrial dysfunction mediated by excitatory amino acid in the CNS, Neurochem. Int. 50 (2007) 418-426.

[8] A. Domney, J. Feldon, The disruption of prepulse inhibition by social isolation in the wistar rat: How robust is the effect? Pharmacol. Biochem. Behav. 59 (1998) 883-890.

[9] Y. Enokido, E. Suzuki, K. Iwasawa, K. Namekata, H. Okazawa, H. Kimura, Cystathionine $\beta$-synthase, a key enzyme for homocysteine metabolism, is preferentially expressed in the radial glia/astrocyte lineage of developing mouse CNS, FASEB J. 19 (2005) 1854-1856.

[10] J. D. Finkelstein, Inborn errors of sulfer-containing amino acid metabolism, J.Nutr. 136 (2006) 1750S-1754S.

[11] K. C. F. Fone, M. V. Porkess, Behavioral and neurochemical effects of post-weaning social isolation in rodents-Relevance to developmental neuropsychiatric disorders, Neurosci. Biobehav. Rev. 32 (2008) 1087-1102.

[12] K. C. F. Fone, K. Shalders, Z. D. Fox, R. Arthur, C. A. Marsden, Increased $5-\mathrm{HT}_{2 \mathrm{C}}$ receptor responsiveness occurs on rearing rats in social isolation, Psychopharmacology (Ber1.) 123 (1996) 346-352. 
[13] S. Fukada, Y. Shimada, T. Morita, K. Sugiyama, Suppression of methionine-induced hyperhomocysteinemia by glycine and serine in rats, Biosci. Biotechnol. Biochem. 70 (2006) 2403-2409.

[14] M. K. Harte, S. B. Powell, N. R. Swerdlow, M. A. Geyer, G. P. Reynolds, Deficit in parvalbumin and calbindin immunoreactive cells in the hippocampus of isolation reared rats, J. Neural Transm. 114 (2007) 893-898.

[15] C. A. Heidbreder, I. C. Weiss, A. M. Domeney, C. Pryce, J. Homberg, G. Hedou, J. Feldon, M. C. Moran, P. Nelson, Behavioral, neurochemical and endocrinological characterization of the early social isolation syndrome, Neuroscience 100 (2000) 749-768.

[16] C. Heim, C. B. Nemeroff, The role of childhood trauma in the neurobiology of mood and anxiety disorders: Preclinical and clinical studies, Biol. Psychiatry 49 (2001) 1023-1039.

[17] Y. Hirabayashi, S. Furuya, Roles of L-serine and sphingolipid synthesis in brain development and neuronal survival, Prog. Lipid Res. 47 (2008) 188-203.

[18] N. T. T. Huong, Y. Murakami, M. Tohda, H. Watanabe, K. Matsumoto, Social isolation stress-induced oxidative damage in mouse brain and its modulation by majonoside-R2, a Vietnamese ginseng saponin, Biol. Pharm. Bull. 28 (2005) 1389-1393.

[19] J. Jaeken, M. Detheux, L. Van Maldergem, M. Foulon, H. Carchon, E. Van Schaftingen, 3-Phosphoglycerate dehydrogenase deficiency: an 
inborn error of serine biosynthesis, Arch. Dis. Child. 74 (1996) 542-545.

[20] T. Koutoku, H. Takahashi, S. Tomonaga, D. Oikawa, S. Saito, T. Tachibana, L. Han, K. Hayamizu, D. M. Denbow, M. Furuse, Central administration of phosphatidylserine attenuates isolation stress-induced behavior in chicks, Neurochem. Int. 47 (2005) 183-189.

[21] A. Kumar, S. K. Kulkarni, Protective effect of BR-16A, a polyherbal preparation against social isolation stress: Possible GABAergic mechanism, Phytother. Res. 20 (2006) 538-541.

[22] J. B. Levine, R. M. Youngs, M. L. Macdonald, M. Chu, A. D. Leeder, F. Berthiaume, C. Konradi, Isolation rearing and hyperlocomotion are associated with reduced immediate early gene expression levels in the medial prefrontal cortex, Neuroscience 145 (2007) $42-55$.

[23] J. Mitoma, S. Furuya, Y. Hirabayashi, A novel metabolic communication between neurons and astrocytes: non-essential amino acid L-serine released from astrocytes is essential for developing hippocampal neurons, Neurosci. Res. 30 (1998) $195-199$.

[24] J. Mitoma, T. Kasama, S. Furuya, Y. Hirabayashi, Occurrence of an unusual phospholipid, phosphatidyl-L-threonine, in cultured hippocampal neurons, J. Biol. Chem. 273 (1998) 19363-19366.

[25] M. G. Nunzi, F. Milan, D. Guidolin, G. Toffano, Dendritic spine loss in hippocampus of aged rats. Effect of brain phosphatidylserine administration, Neurobiol. Aging 8 (1987) 501-510.

[26] N. C. A. Schrijver, P. N. Pallier, V. J. Brown, H. Wurbel, Double 
dissociation of social and environmental stimulation on spatial learning and reversal learning in rats, Behav. Brain Res. 152 (2004) 307-314.

[27] M. Serra, M. G. Pisu, M. Littera, G. Papi, E. Sanna, F. Tuveri, L. Usala, R. H. Purdy, G. Biggio, Social isolation-induced decreases in both the abundance of neuroactive steroids and $\mathrm{GABA}_{\mathrm{A}}$ receptor function in rat brain, J. Neurochem. 75 (2000) $732-740$.

[28] M. Serra, M. G. Pisu, M. C. Mostallino, E. Sanna, G. Biggio, Changes in neuroactive steroid content during social isolation stress modulate $\mathrm{GABA}_{\mathrm{A}}$ receptor plasticity and function, Brain Res. Rev. 57 (2008) $520-530$.

[29] K. Shigemi, Y. Tsuneyoshi, K. Hamasu, L. Han, K. Hayamizu, D. M. Denbow, M. Furuse, L-Serine induces sedative and hypnotic effects acting at $\mathrm{GABA}_{\mathrm{A}}$ receptors in neonatal chicks, Eur. J. Pharmacol. 599 (2008) 86-90.

[30] A. B. Silva-Gomez, D. Rojas, I. Juarez, G. Flores, Decreased dendritic spine density on prefrontal cortical and hippocampal pyramidal neurons in postweaning social isolation rats, Brain Res. 983 (2003) 128-136.

[31] R. Surtees, A. Bowron, J. Leonard, Cerebrospinal fluid and plasma total homocysteine and related metabolites in children with cystathionine beta-synthase deficiency: the effect of treatment, Pediatr. Res. 42 (1997) $577-582$.

[32] S. Suzuki, H. Yamatoya, M. Sakai, A. Kataoka, M. Furushiro, S. Kudo, Oral administration of soybean lecithin transphosphatidylated 
phosphatidylserine improves memory impairment in aged rats, Nutr. Neurosci. 131 (2001) 2951-2956.

[33] G. B. Varty, C. A. Marsden, G. A. Higgins, Reduced synaptophysin immunoreactivity in the dentate gyrus of prepulse inhibition-impaired isolation-reared rats, Brain Res. 824 (1999) 197-203.

[34] H. Yamane, M. Asechi, Y. Tsuneyoshi, D. M. Denbow, M. Furuse, Central L-cysteine induces sleep, and D-cycteine induces sleep and abnormal behavior during acute stress in neonatal chicks, Anim. Sci. J. $80(2009) 428-432$.

[35] M. Yamasaki, K. Yamada, S. Furuya, J. Mitoma, Y. Hirabayashi, M. Watanabe, 3-Phosphoglycerate dehydrogenase, a key enzyme for L-serine biosynthesis, is preferentially expressed in the radial glia/astrocyte lineage and olfactory ensheathing glia in the mouse brain, J. Neurosci. 21 (2001) 7691-7704.

[36] K. Yoshida, S. Furuya, S. Osuka, J. Mitoma, Y. Shinoda, M. Watanabe, N. Azuma, H. Tanaka, T. Hashikawa, S. Itohara, Y. Hirabayashi, Targeted disruption of the mouse 3-phosphoglycerate dehydrogenase gene causes severe neurodevelopmental defects and results in embryonic lethality, J. Biol. Chem. 279 (2004) 3573-3577. 


\section{Figure Legends}

Fig. 1. Effect of L-serine on the locomotor activity of isolated rats in their home cages during the dark period. Results are expressed as means \pm S.E.M. The number of rats used in each group was 7 .

Fig. 2. Effect of L-serine on (A) distance of path (B) moving time (C) movement speed, and (D) frequency of rearing behavior of rats in an open field during a $30 \mathrm{~min}$ period. Results are expressed as means \pm S.E.M. The number of rats used in each group was 7. Different letters indicated significant difference at $\mathrm{P}<0.05$.

Fig.3. Effect of L-serine on the concentration of L-serine and L-cystathionine in the cerebral cortex (upper panel) or hippocampus (lower panel). Results are expressed as means \pm S.E.M. The number of samples used for analysis was 6-7. Group with different letters are significantly different $(\mathrm{P}<0.05)$. 


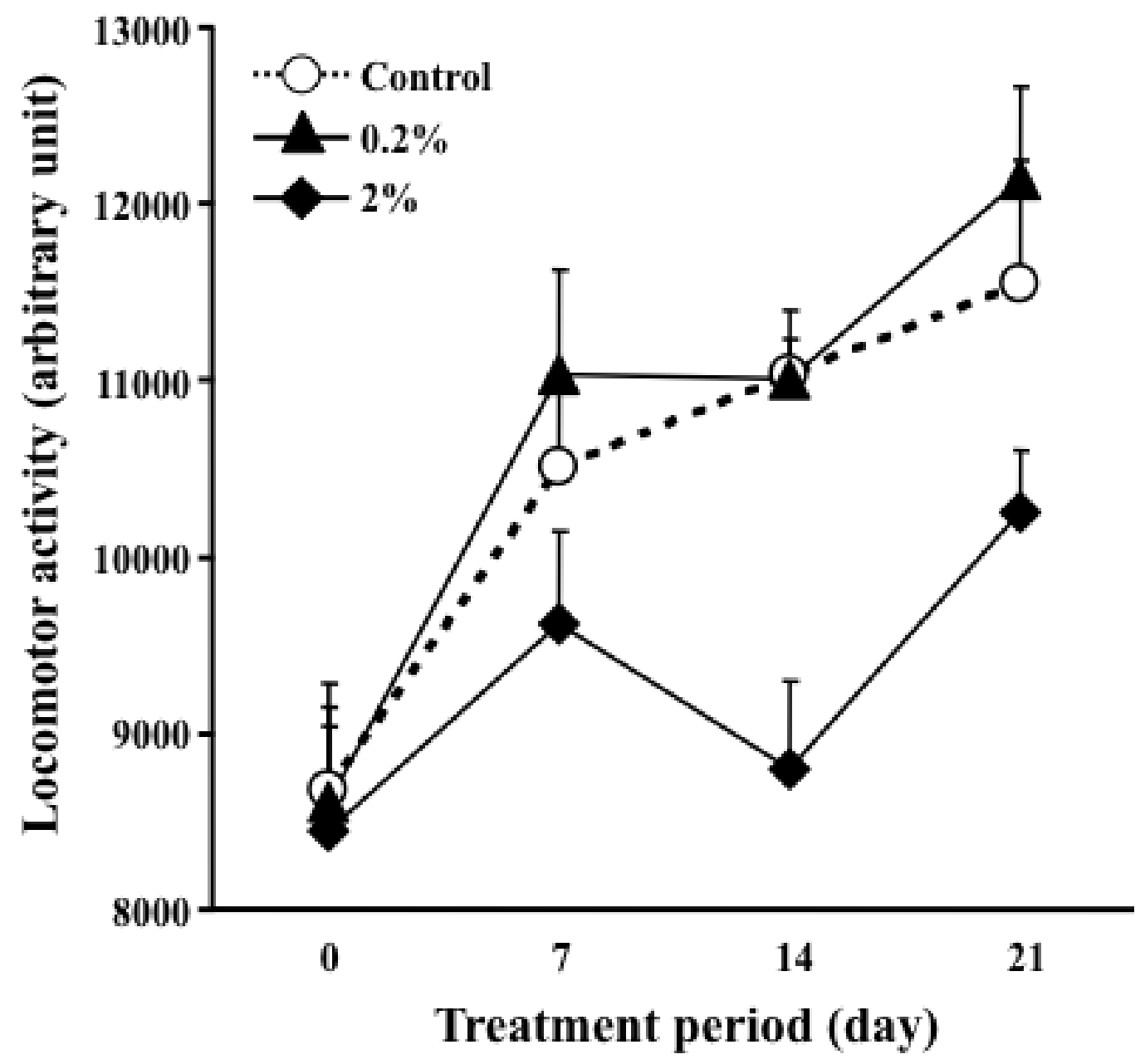

Fig. 1. Shigemi et al. 

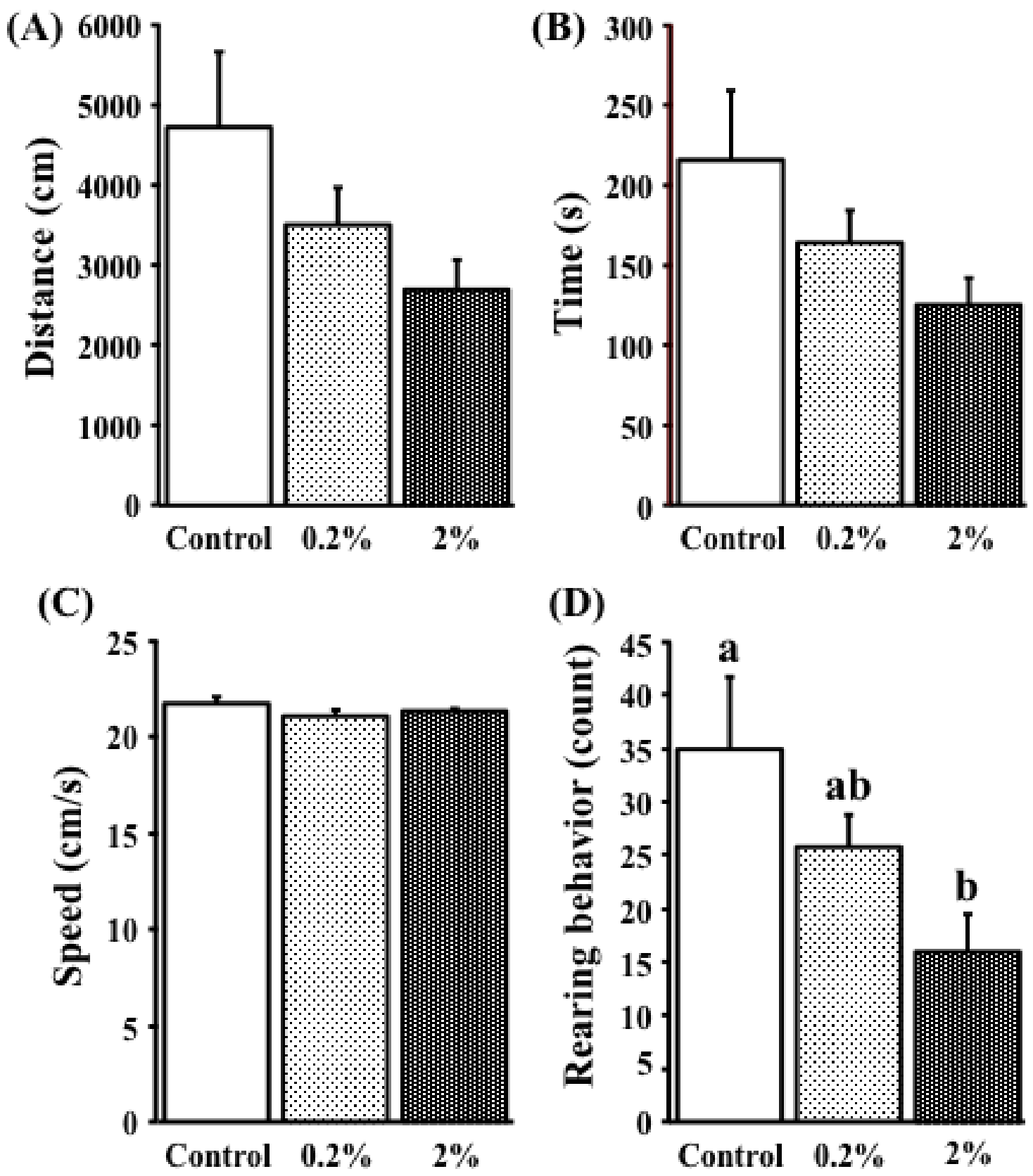

(D)

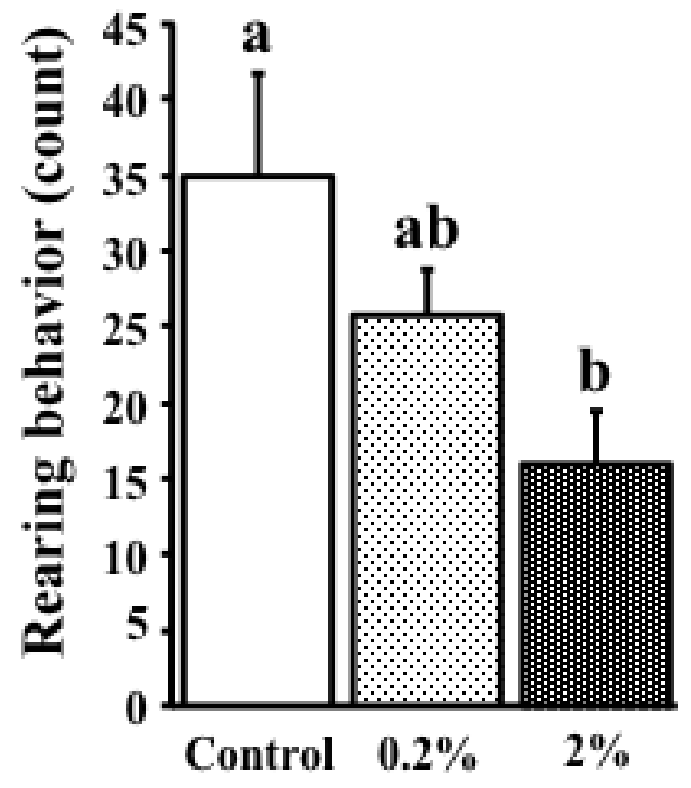

Fig. 2. Shigemi et al. 


\section{Cerebral cortex}

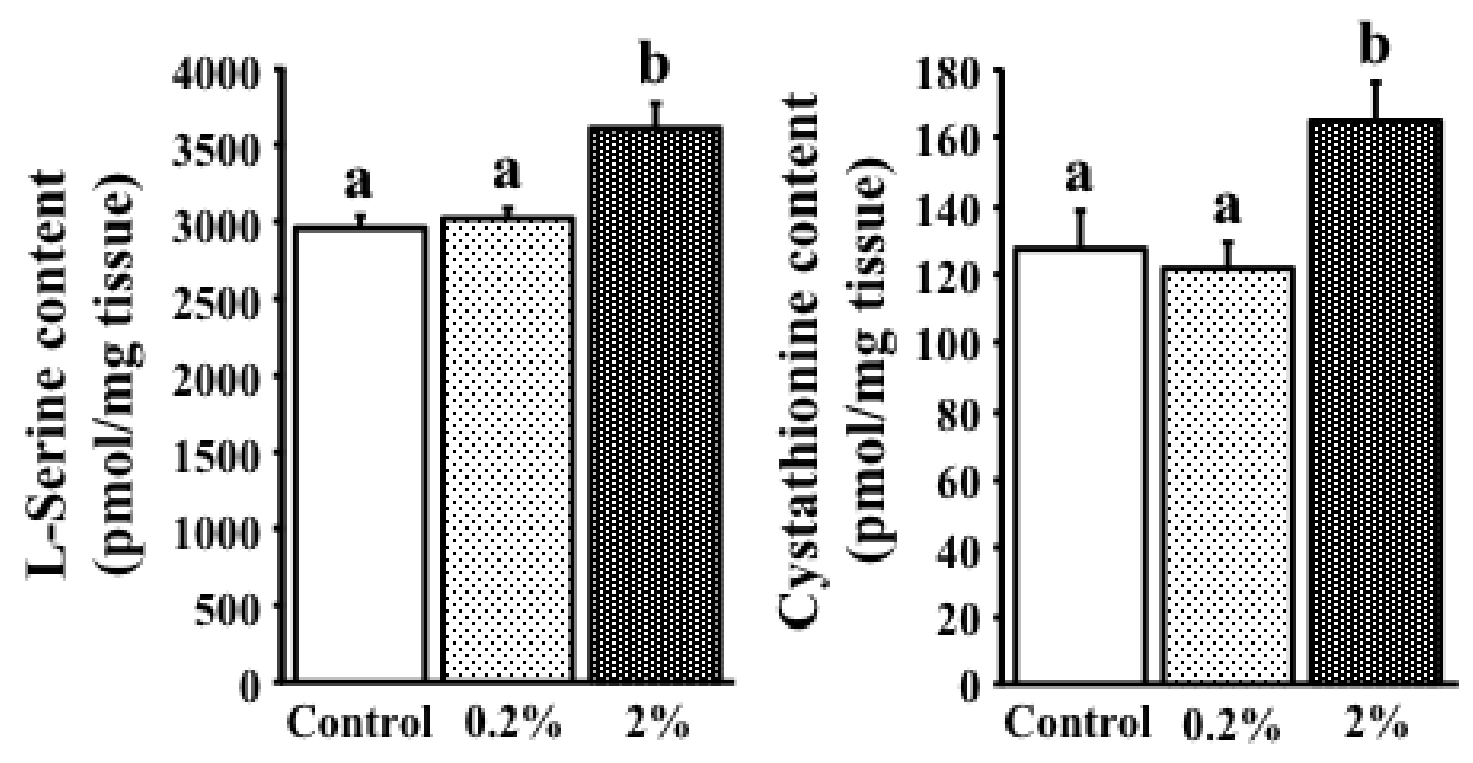

Hippocampus
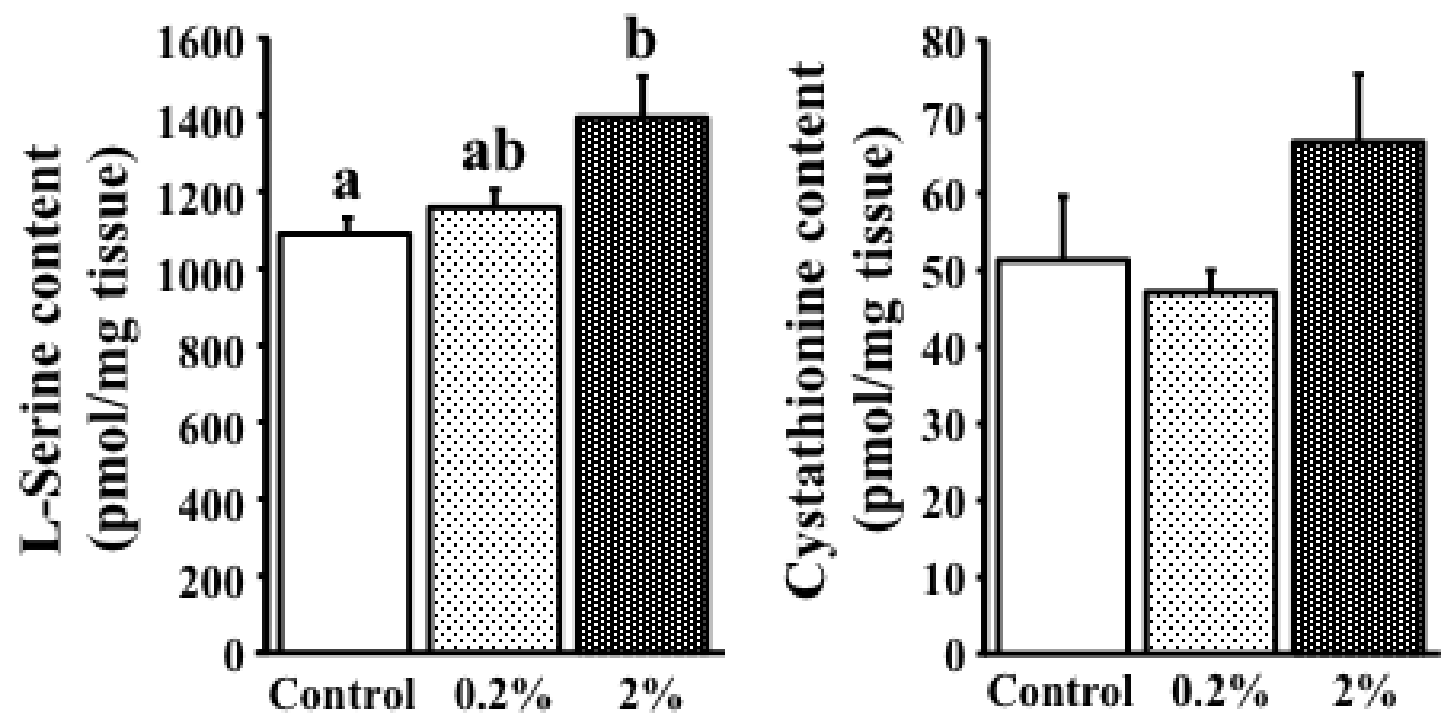

Fig. 3. Shigemi et al. 